\title{
Periodicity Dependence of Magnetic Anisotropy and Magnetization of FeCo Heterostructure
}

\author{
Miyoung Kim* \\ Department of Nano Physics, Sookmyung Women's University, Seoul 04310, Korea
}

(Received 29 January 2016, Received in final form 23 February 2016, Accepted 23 February 2016)

\begin{abstract}
The magnetic anisotropy energy (MAE) and the saturation magnetization $\mathrm{B}_{\mathrm{s}}$ of (110) $\mathrm{Fe}_{\mathrm{n}} \mathrm{Co}_{\mathrm{n}}$ heterostructures with $\mathrm{n}=1,2$, and 3 are investigated in first-principles within the density functional theory by using the precise full-potential linearized augmented plane wave (FLAPW) method. We compare the results employing two different exchange correlation potentials, that is, the local density approximation (LDA) and the generalized gradient approximation (GGA), and include the spin-orbit coupling interaction of the valence states in the second variational way. The MAE is found to be enhanced significantly compared to those of bulk Fe and Co and the magnetic easy axis is in-plane in agreement with experiment. Also the MAE exhibits the in-plane angle

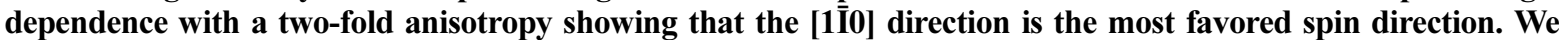
found that as the periodicity increases, (i) the saturation magnetization $B_{s}$ decreases due to the reduced magnetic moment of $\mathrm{Fe}$ far from the interface, (ii) the strength of in-plane preference of spin direction increases yielding enhancement of MAE, and (iii) the volume anisotropy coefficient decreases because the volume increase outdo the MAE enhancement.
\end{abstract}

Keywords : first-principles calculations, electronic structure, magnetic anisotropy, magnetization

\section{Introduction}

$\mathrm{Fe}$ and $\mathrm{Co}$ are the representative transition metals exhibiting ferromagnetic ordering solely in their bulk forms, which makes them the major ingredients in designing new materials for the high density storage media. In this application, the novel materials with an enhaced saturation magnetization and the perpendicular magnetocrystalline anisotropy (MCA) with the large magnetic anisotropy energy (MAE) have been intensively searched for many decades aiming for the further increasing of recording density above the superparamagnetic limit $[1,2]$. To obtain the high saturation magnetization, various efforts such as making the alloys of Fe or Co with the $4 \mathrm{~d}$ - or $5 \mathrm{~d}$ - transition metals to invoke the proximity effect or doping the rare earth elements pocessing high magnetic moment have been made whereas the binary alloy $\mathrm{Fe}_{1-\mathrm{x}} \mathrm{Co}_{\mathrm{x}}$ with the Co concentration of $\sim 35 \%$ is predicted to have the highest value of $\sim 2.45 \mathrm{~T}$ as predicted by the Slater-Pauling curve [3]. A giant magnetic

(C)The Korean Magnetics Society. All rights reserved.

*Corresponding author: Tel: +82-2-2077-7077

Fax: +82-0303-0799-0362, e-mail: mykim.nu@gmail.com moment up to $3.2 \mu_{\mathrm{B}}$ for $\mathrm{Fe}$ was reported in a quasistable $\mathrm{Fe}_{16} \mathrm{~N}_{2}$ suggesting a huge magnetization while the effect of nitrogen in enhancing the saturatuin magnetization is still controversal [4]. On the other hand, the high MAE can be achieved by introducing the heavy $5 \mathrm{~d}$ transition metals with the large spin-orbit coupling strength such as $\mathrm{Pt}$ [5]. Another efficient way to improve MAE is to lower the lattice symmetry by creating the tetragonal distortion or artificial heterostructures such as superlattices or multilayers, which is known to increase MAE of a few meV in bulk by more than a thousand times.

The phenomenological expression of MCA can be written by the anisotropy coefficients and the polar and azimuthal angles, $\theta$ and $\phi$. For the (001) plane of cubic symmetry with four fold rotational symmetry, for example, MCA can be written as [6]

$$
E_{M C A} \cong-K_{1}^{(2)} \cos ^{2}(\theta)+K_{2}^{(4)} \sin ^{4}(\theta) \cos (4 \phi)+\mathrm{O}\left(\alpha^{6}\right),
$$

where $K_{1}{ }^{(2)}$ and $K_{2}{ }^{(4)}$ are the anisotropy coefficients and $\alpha$ is the direction cosine of the magnetic moment with respect to the coordinate axes. One can see that the leading order term comes from the second order direction cosines and depends only on $\theta$. The $\phi$ dependence, exhibiting a four fold in-plane symmetry, comes from the 
fourth order direction cosines which is negligible compared to the second order term. For the (110) plane which has two-fold rotational symmetry, however, the phenominological MCA should be written as

$$
E_{M C A} \cong K_{1}^{(2)} \cos ^{2}(\theta)+K_{2}^{(2)} \sin ^{2}(\theta) \cos ^{2}(\phi)+\mathrm{O}\left(\alpha^{4}\right) .
$$

Here, the $\phi$ dependence appears in one of the leading order terms, implying that the in-plane dependence of MCA is no longer negligible. Moreover, one can obtain the anisotropy coefficients by fitting the in-plane dependent MCA using the equation (2).

In this work, we carried out first-principles calculations for $\mathrm{Fe}_{\mathrm{n}} \mathrm{Co}_{\mathrm{n}}$ (110) heterostructures with $\mathrm{n}=1,2$, and 3 within the density functional theory to investigate the magnetic properties including the magnetic anisotropy energy (MAE) and the magnetization. The in-plane dependence of MAE is calculated and the anisotropy coefficients are determined. Our main interest is to find how the results depend on the periodicity, the tetragonal distortion, and the exchange correlation potentials.

\section{Methodology}

We considered three heterostructures of $\mathrm{Fe}_{n} \mathrm{Co}_{\mathrm{n}}$ (110) with $\mathrm{n}=1,2$, and 3 where $\mathrm{n}$ layers of bcc $\mathrm{Fe}$ and $\mathrm{Co}$ (110) planes are alternatively stacked along the [110] direction as shown in Figure 1. The equilibrium c/a ratio of the resulting body-centered tetragonal lattice is determined by the total energy calculations while the (110) inplane lattice constant $a$ is fixed as the value of bulk bcc Fe. We performed electronic structure calculations within the density functional theory by using the all-electron full-potential augmented plane-wave (FLAPW) method [7]. For the exchange-correlational potential, we employed two different approaches, that is, the local density approximation (LDA) and the generalized gradient approximation (GGA) [8] for comparison. An energy cut off of $220 \mathrm{eV}$ is used for the plane-wave basis in the interstitial region and the lattice harmonics with angular momentum $l$ up to 8 are used for the wave functions, the
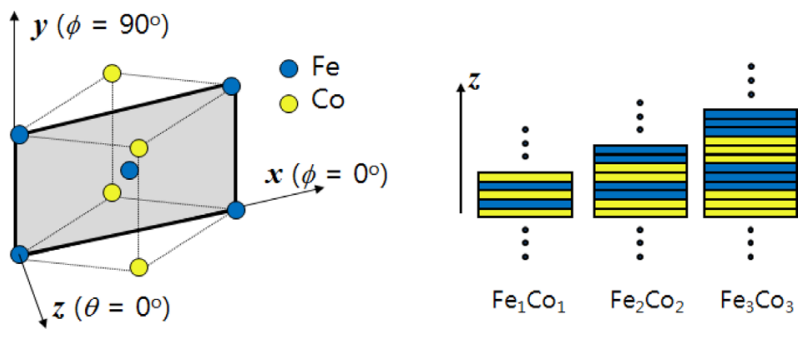

Fig. 1. (Color online) Schematic figure for the heterostructures of $\mathrm{Fe}_{\mathrm{n}} \mathrm{Co}_{\mathrm{n}}(110)$ where $\mathrm{n}=1,2$, and 3 . charge density and the potentials within the muffin-tin (MT) spheres of radii $\mathrm{R}_{\mathrm{MT}}=2.30 \mathrm{a}$. $\mathrm{u}$. for both $\mathrm{Fe}$ and Co atoms. The spin-orbit coupling (SOC) plays a critical role in determining the magnetocrystalline anisotropy and thus the MAE. We include the SOC fully to describe the core states and in a second variational way to obtain the valence states [9]. The SOC Hamiltonian matrix elements are calculated by integrating the radial derivatives of the potentials over the MT region of Fe and Co atoms while the interstitial contributions are neglected.

To determine the new SOC induced occupied states and Fermi levels, we adopt the state tracking method which is known to induce a fast convergence of MAE with respect to the number of $\mathrm{k}$ points by effectively reducing the fluctuation [10]. The MAE is defined as the difference in the total energies for two directions of magnetization; the in-plane $\left(\theta=0^{\circ}\right)$ and the perpendicular $\left(\theta=90^{\circ}\right)$ directions to the given surface. If the near-degenerate states are located close to the Fermi level, an instability of MAE often occurs because two different sets of calculations which are carried out separately can have different occupied states and thus notably modified Fermi surfaces. To further suppress these remaining uncertainties, we used the torque method [11] which reduces two distinct sets of total energy calculations into one shot calculation of the total energy and its derivative with respect to the polar angle $\theta$, evaluated at $\theta=45^{\circ}$, as can be seen in the next section.

\section{Results and Discussion}

The tetragonal distortion (or $c / a$ ratio) was reported to be crucial to determine the MAE for the FeCo system [12]. Therefore, we first performed total energy calculations to find the equilibrium $c / a$ ratio for all heterostructures while the in-plane (110) lattice is fixed as the bcc Fe lattice constant. We show our calculated total energy and saturation magnetization, $\mathrm{B}_{\mathrm{s}}$, for various $c / a$ ratios in Figure 2 in LDA (a) and in GGA (b). As the $c$-lattice expands, the total magnetization per volume $B_{s}$ monotonically decreases for all three heterostructures in both LDA and GGA. This is because while the total magnetic moment is slightly enhanced as $c / a$ ratio increases due to the band narrowing effect, the increase of total magnetic moment is not big enough to compensate for the volume increase. The equilibrium $c / a$ ratio is found to be 0.933 , 0.932 , and 0.928 in LDA which results in the periodicities of 3.77, 7.53, and $11.27 \AA$ in length along the $c$-direction for $\mathrm{n}=1,2$, and 3, respectively. In GGA, on the other hand, we found that the equilibrium $c / a$ ratio is 0.980 for all three systems which results in the periodicities of 3.97, 

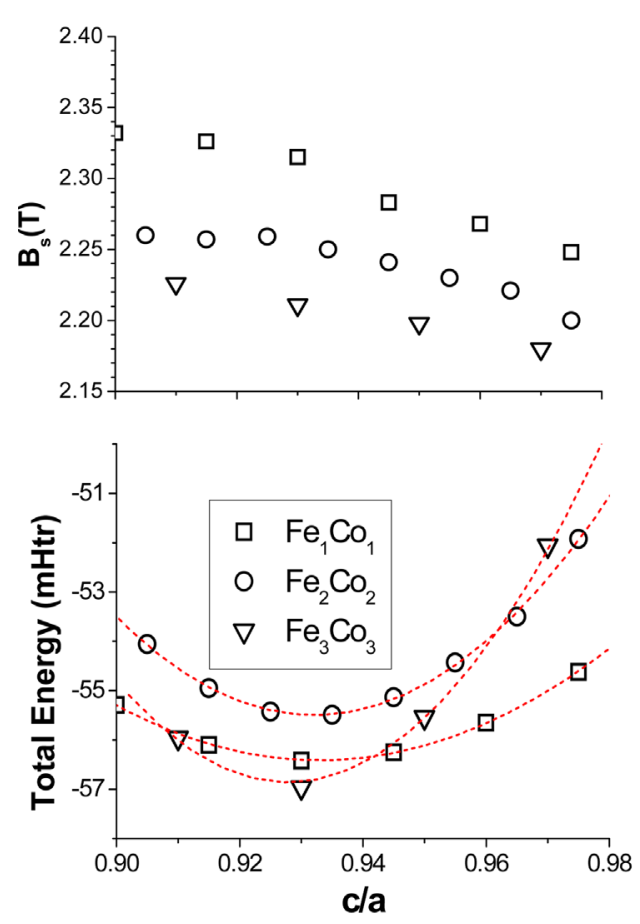

(a)
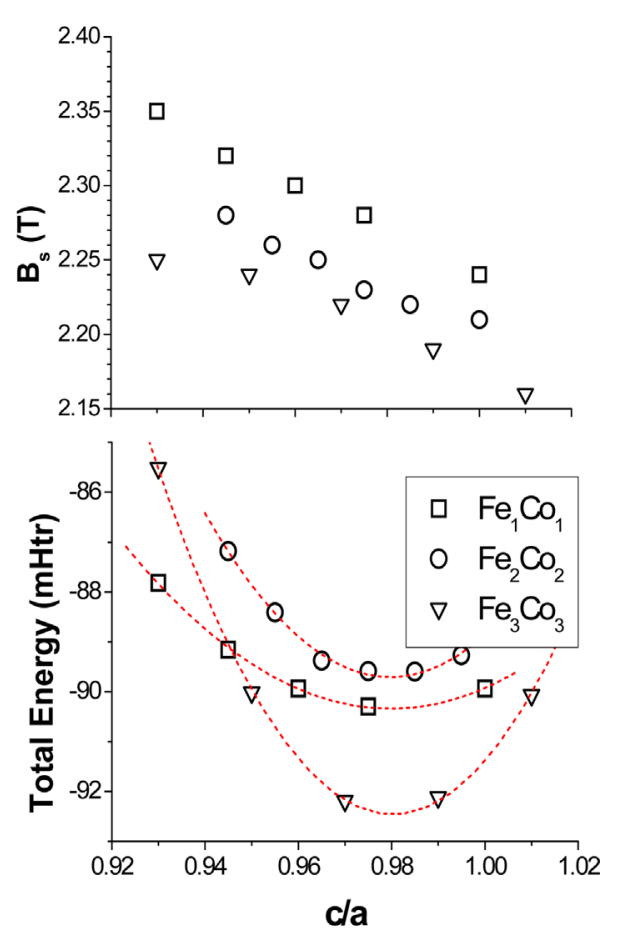

(b)

Fig. 2. (Color online) Calculated total energy (in $\mathrm{mHtr}$ ) and spin saturation magnetization, $\mathrm{B}_{\mathrm{s}}$ (in $\mathrm{T}$ ) as the functions of the tetragonal distortion ( $c / a$ ratio) for $\mathrm{Fe}_{\mathrm{n}} \mathrm{Co}_{\mathrm{n}}(110)$ heterostructures for $\mathrm{n}=1$ (squares), 2 (circles), and 3 (triangles) within LDA (a) and GGA (b).

7.94, and $11.91 \AA$ in length for $n=1,2$, and 3, respectively. Note that the periodicity in length can be obtained by multiplying the $c / a$ ratio, the in-plane lattice, and the value n. As is well known, the GGA gives bigger values of the lattice constants and the magnetic moments compared to those of LDA. The large GGA magnetic moment is partly due to the volume expansion and also due to the enhanced exchange potential. We found that GGA moments are bigger than LDA moments for the present material but the magnetic moment enhancement does not outdo the volume expansion. As a results, $\mathrm{B}_{\mathrm{s}}$ values at the equilibrium $c / a$ ratio in GGA are found to be slightly smaller than those in LDA for all three heterostructures of $n=1$, 2 , and 3 as can be seen in Fig. 2. The $B_{\text {s }}$ values at the equilibrium $c / a$ ratio are shown in Table 1.

The Fig. 2 also shows that, as the periodicity increases, the calculated saturation magnetization $B_{s}$ decreases in agreement with experiment [13]. The decrease of $B_{s}$ for large periodicity is mostly due to the Fe spin moment which is reduced as it goes far from the Co interface; in GGA, the spin moments are $2.58 \mu_{\mathrm{B}}$ for the interface Fe and $2.44 \mu_{\mathrm{B}}$ for the next interface $\mathrm{Fe}$ atom for $\mathrm{n}=3$, which are significantly reduced from the Fe moment 2.65 $\mu_{\mathrm{B}}$ for $\mathrm{n}=1$. In contrast, the Co moment is relatively less influenced by the atomic environment; 1.87 and $1.85 \mathrm{~m}_{\mathrm{B}}$ for $n=3$ and 1, respectively. As a result, the calculated saturation magnetizations are in the ranges of $2.32 \sim 2.10 \mathrm{~T}$ as shown in Table 1 (a), which is underestimated compared to the experimental $B_{\mathrm{s}}$ around 2.40 2.30 $\mathrm{T}$ for the periodicities corresponding to the present system. For the saturation magnetizations, we included both spin and orbital magnetic moments in our calculation. The orbital angular moments are known to be underestimated by DFT calculations compared to the experimental values: we obtained the calculated orbital moments 0.05 and 0.08 $\mu_{\mathrm{B}}$ for $\mathrm{Fe}$ and $\mathrm{Co}$, respectively, while experimental values are $0.09(\mathrm{Fe})$ and $0.16 \mu_{\mathrm{B}}(\mathrm{Co})$ [14]. If we adopt the

Table 1. The calculated saturation magnetization $B_{s}$ in Tesla at the equilibrium $c / a$ ratio. The column (a) is the result from the calculated spin and orbital moments; the column (b) is from the calculated spin moments and the experimental orbital moments. Experimental $\mathrm{B}_{\mathrm{s}}$ is given for comparison from the reference [13].

\begin{tabular}{|c|c|c|c|c|c|}
\hline \multirow{2}{*}{ system } & \multicolumn{2}{|c|}{$\mathrm{B}_{\mathrm{s}}$ in LDA $(\mathrm{T})$} & \multicolumn{2}{|c|}{$\mathrm{B}_{\mathrm{s}}$ in GGA (T) } & \multirow{2}{*}{$\begin{array}{c}\mathrm{B}_{\mathrm{s}} \text { in experiment }(\mathrm{T}) \\
{[13]}\end{array}$} \\
\hline & (a) & (b) & (a) & (b) & \\
\hline $\mathrm{Fe}_{1} \mathrm{Co}_{1}$ & 2.32 & 2.39 & 2.27 & 2.33 & 2.38 \\
\hline $\mathrm{Fe}_{2} \mathrm{Co}_{2}$ & 2.27 & 2.34 & 2.24 & 2.30 & 2.35 \\
\hline $\mathrm{Fe}_{3} \mathrm{Co}_{3}$ & 2.22 & 2.28 & 2.21 & 2.27 & 2.30 \\
\hline
\end{tabular}




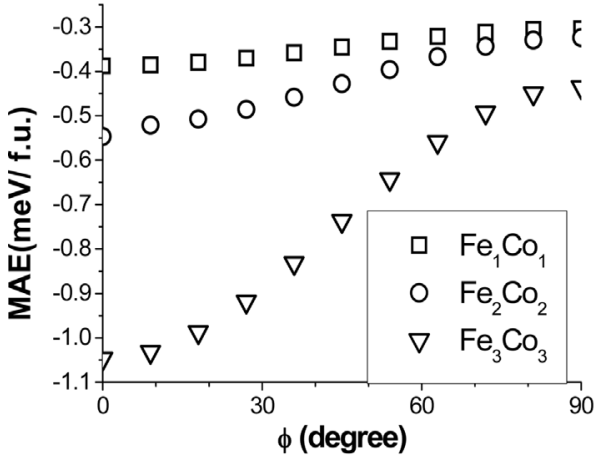

(a)

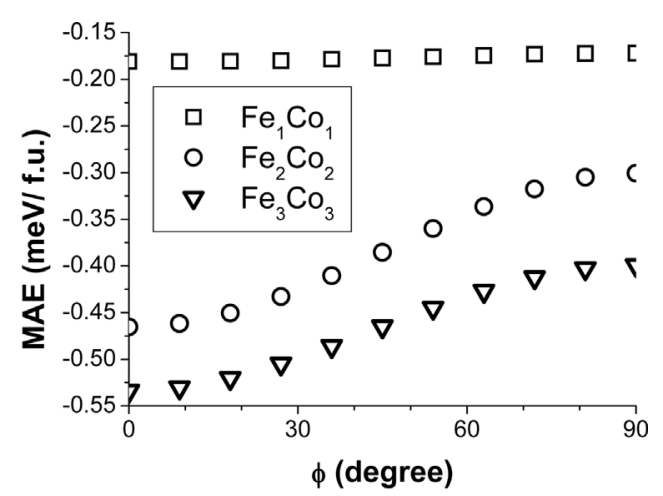

(b)

Fig. 3. Calculated magnetic anisotropy energy (MAE) per formula unit within (a) LDA and (b) GGA, as a function of the in-plane angle $\phi$ measured from the direction of the in-plane nearest neighbor atom.

experimental orbital moments instead of the calculated values, $B_{\mathrm{s}}$ enhances to $2.39,2.34$, and $2.28 \mathrm{~T}$ in LDA and 2.33, 2.30, and $2.27 \mathrm{~T}$ in GGA, for $\mathrm{n}=1,2$, and 3 , respectively, as shown in Table 1 (b) which gives better agreement with experiment.

We calculated the MAE for each heterostructures with the equilibrium $c / a$ value and showed the result as a function of $\phi$ in Fig. 3 for LDA (a) and GGA (b). The inplane angle $\phi$ is measured from the direction along [1 $\mathrm{I} 0]$ in the [110] plane. The magnetic anisotropy energy MAE is defined as the total energy difference between the inplane and the perpendicular magnetic orientations. Therefore, the positive (negative) MAE value indicates that the magnetic moments prefer to align perpendicular (in-plane) to the surface. While the MAE is given as a function of the azimuthal angle $\phi$, for the surface with four-fold rotational symmetry in cubic lattice, the $\phi$ dependence is negligible and the MAE is generally calculated for a fixed $\phi$ value as mentioned above. However, the present heterostructures of (110) plane have the two-fold rotational symmetry and thus the in-plane dependence of MAE come from the leading order terms. Using the MCA expression given in equation (2), the MAE $\Delta E$ for (110) surface can be written as

$$
\Delta E=E\left(\theta=90^{\circ}, \phi\right)-E\left(\theta=0^{\circ}\right) \cong-K_{1}^{(2)}+K_{2}^{(2)} \cos ^{2}(\phi),
$$

where the anisotropy constants $K_{1}^{(2)}$ and $K_{2}^{(2)}$ come from the same order. Therefore, this expression indicates that the MAE of (110) surface exhibits a two fold in-plane anisotropy. This equation also confirms that the torque method is valid for MAE calculation. As can be seen from Fig. 3, all systems have the negative values of MAE implying that the in-plane spin direction is favored over the perpendicular direction to the (110) surface. The maximum MAE values in magnitude are found at $\phi=0$, indicating that the in-plane easy axis is along [1] 0$]$ which is the direction of the in-plane third nearest neighbor atom. Also all systems exhibit the two fold in-plane anisotropy that is well fitted by a function $-K_{1}^{(2)}+$ $K_{2}{ }^{(2)} \cos ^{2}(\phi)$ as predicted in the equation (3). The anisotropy constants obtained by the fitting are given in Table 2 for LDA and GGA. The anisotropy constant $K_{2}{ }^{(2)}$ gives the strength of the in-plane anisotropy while $-K_{1}{ }^{(2)}+K_{2}{ }^{(2)}$ indicates the strength of the perpendicular anisotropy. Results show that the strength of the in-plane anisotropy is the same order of magnitude as the perpendicular anisotropy for $\mathrm{n}=2$ and 3 while the in-plane anisotropy is negligibly smaller than the perpendicular anisotropy for $n$ $=1$. For fixed $\phi$, the magnitude of MAE increases as the periodicity increases which means that the in-plane preference of spin direction is enhanced. This is interesting result because one may usually expect the increase of periodicity to invoke the bulk property which, in this case, may be a suppression of MAE. The Fig. 3 shows that the overall tendency of the MAE dependence on the angle $\phi$ and the periodicity is similar for the LDA and the GGA. The calculated anisotropy constants in Table 2, however, exhibit the dependence on the exchange correlation potential functional, especially for $\mathrm{n}=1$ and 3. This

Table 2. The calculated magnetic anisotropy constants $K_{1}^{(2)}$ and $K_{2}^{(2)}$ in meV/f.u. which are obtained by fitting the calculated MAE in LDA and GGA by a function $-K_{1}^{(2)}+$ $K_{2}^{(2)} \cos ^{2}(\phi)$.

\begin{tabular}{cccccc}
\hline \hline \multirow{2}{*}{ system } & \multicolumn{2}{c}{$K_{1}^{(2)}(\mathrm{meV} /$ f.u. $)$} & & \multicolumn{2}{c}{$K_{2}^{(2)}(\mathrm{meV} /$ f.u. $)$} \\
\cline { 2 - 3 } \cline { 5 - 6 } & LDA & GGA & & LDA & GGA \\
\hline $\mathrm{Fe}_{1} \mathrm{Co}_{1}$ & 0.30 & 0.17 & & -0.09 & -0.01 \\
$\mathrm{Fe}_{2} \mathrm{Co}_{2}$ & 0.31 & 0.30 & & -0.45 & -0.46 \\
$\mathrm{Fe}_{3} \mathrm{Co}_{3}$ & 0.44 & 0.40 & & -0.61 & -0.13 \\
\hline
\end{tabular}




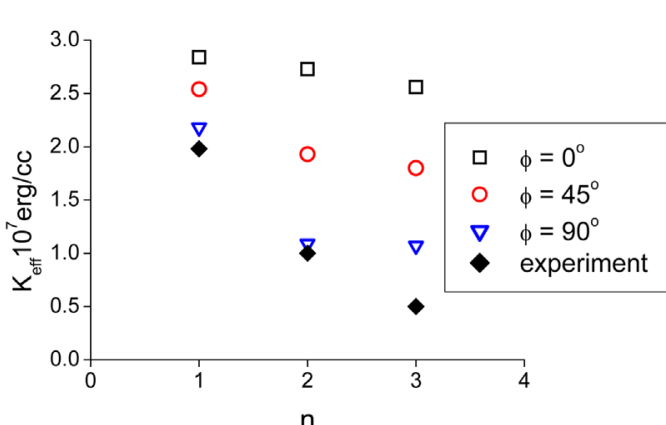

(a)

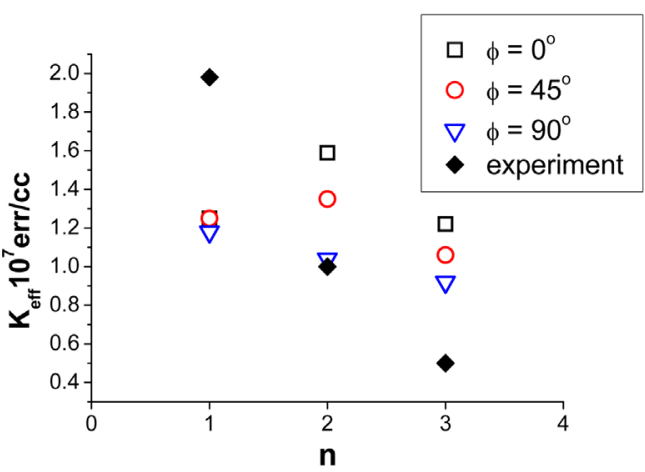

(c)

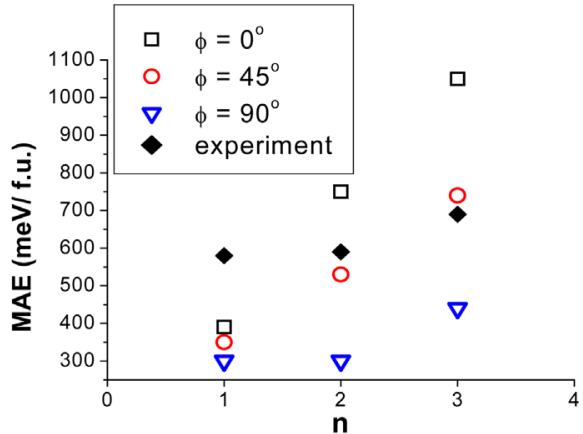

(b)

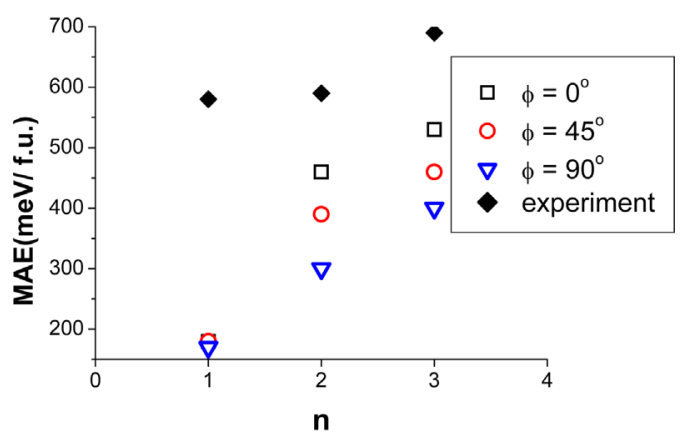

(d)

Fig. 4. (Color online) Calculated and experimental magnetic anisotropy coefficients $\mathrm{K}_{\text {eff }}$ (in $10^{7}$ erg/cc) and magnetic anisotropy energies (MAE) (in meV/f.u.) as the functions of periodicity $\mathrm{n}$ of $\mathrm{Fe}_{\mathrm{n}} \mathrm{Co}_{\mathrm{n}}$ (110) within LDA ((a) and (b)) and GGA ((c) and (d)). Calculated results for three azimuthal angles, that is, $\phi=0^{\circ}$ (in squares), $45^{\circ}$ (in circles), and $90^{\circ}$ (in triangles) are given to show their in-plane dependences.

difference might be partly from the difference in the potentials as well as the $c / a$ ratio.

The Fig. 4 shows our calculated results of MAE together with the experimental results [15]. As explained above, our calculated results have the in-plane angle dependence while the experiment is given at a fixed inplane angle. Therefore, our MAE at $\phi=0^{\circ}$ and $\phi=90^{\circ}$ show the maximum and minimum bound of the MAE. The figure clearly shows that the overall trends of the periodicity dependence in the calculational and experimental MAE agree each other, that is, while the total MAE increases with increasing periodicity, the anisotropy energy per volume, $\mathrm{K}_{\text {eff }}$, decreases as the periodicity increases. Despite this agreement in the overall behavior, the computational and experimental MAE values show differences - perhaps because of inter-layer mixing, relaxation of the in-plane lattice constant, or local atomic displacements, not concerned in our calculations.

\section{Conclusion}

We showed that the saturation magnetization $\mathrm{B}_{\mathrm{s}}$ of $\mathrm{Fe}_{\mathrm{n}} \mathrm{Co}_{\mathrm{n}}(110)$ heterostructures with $\mathrm{n}=1,2$, and 3 are in the range of 2.31 2.10 $\mathrm{T}$ from the first-principles calculations within the density functional theory. They decrease as the periodicity of the heterostructure increases in agreement with experiment and can be enhanced up to as high as $2.39,2.34$, and $2.28 \mathrm{~T}$ in the LDA adapting the experimental orbital magnetic moments while the GGA gives slightly reduced values due to the bigger lattice constants. All three systems have the in-plane magnetic easy axis and exhibit the in-plane angle dependent MAE with two-fold anisotropy which has the same order of magnitude as the perpendicular anisotropy. The magnitude of MAE increases as the periodicity increases indicating that the in-plane preference of spin moment direction is enhanced. Despite these results are consistent with experiments, the calculated anisotropy constants deviate from the experimental values which might be improved by more realistic simulations including the inter-layer mixing and the optimization of the in-plane lattice constants as well as the internal atomic positions.

\section{Acknowledgements}

This work is supported by the Basic Science Research 
Program through the NRF of Korea funded by the Ministry of Education, Science and Technology (2013R1A1A3013348).

\section{References}

[1] D. Weller and A. Moser, IEEE Trans. Magn. 35, 4423 (1999).

[2] A. J. Minnich, M. S. Dresselhaus, Z. F. Ren, and G. Chen, Energy Environ. Sci. 2, 466 (2009).

[3] R. M. Bozorth, Ferromagnetism, Van Nostrand, New York (1951).

[4] T. K. Kim and M. Takahashi, Appl. Phys. Lett. 20, 492 (1972).

[5] D. Weller, A. Moser, L. Folks, M. E. Best, W. Lee, M. Toney, M. Schwickert, J. U. Thiele, and M. Doerner, IEEE Trans. Magn. 36, 10 (2000).

[6] M. Kim, L. Zhong, and A. J. Freeman, Phys. Rev. B 57, 5271 (1998).

[7] E. Wimmer, H. Krakauer, M. Weinert, and A. J. Freeman,
Phys. Rev. B 24, 864 (1981).

[8] J. P. Perdew, K. Burke, and M. Ernzerhof, Phys. Rev. Lett. 77, 3865 (1996).

[9] A. H. MacDonal, W. E. Pickett, and D. D. Koelling, J. Phys. C: Solid St. Phys. 13, 2675 (1980).

[10] D. S. Wang, R. Wu, and A. J. Freeman, Phys. Rev. B 70, 869 (1993).

[11] X. D. Wang, R. Wu, D. S. Wang, and A. J. Freeman, Phys. Rev. B 54, 61 (1996).

[12] T. Burkert, L.Nordstrom, O. Eriksson, and O. Heinonen, Phys. Rev. Lett. 93, 027203-1 (2004).

[13] V. A. Vas'ko, M. Kim, O. Mryasov, V. Sapozhnikov, M. K. Minor, A. J. Freeman, and M. T. Kief, Appl. Phys. Lett. 89, 902502 (2006).

[14] Landolt-Bornstein, New Series VOL III/19a, edited by H. J. P. Wein, Springer, Berlin (1988).

[15] Experimental data points of $\mathrm{K}_{\mathrm{eff}}$ and MAE are deduced from the Fig. 2 and 3 in Ref [13]. 